\title{
Grand River Hospital's Internet-Based Solutions Empower Patient Managed Care
}

By Claudette DeLenardo

K itchener's Grand River Hospital has launched the first patient portal in North America to provide patients access to their personalized treatment plan, and secure access to their individual care plan.

As part of its ongoing vision to offer state-of-the-art care in a state-of-theart facility, the hospital has launched My CARE Source, a personalized web- based patient portal that provides a patient-focused, family-centred approach to support and disease manage ment for patients with cancer. With increased access to the Internet patients now have the ability to utilize this secure, comprehensive application to enhance their quality of life when and where it meets their needs.

Grand River Hospital has 500 beds offering acute and complex care to the more than 450,000 residents of Waterloo Region. It recently opened the Grand River Regional Cancer Centre, Ontario's first cancer centre fully integrated with an acute care hospital.

My CARE Source is integrated with the hospital health scheduling application and the treatment plan is accompanied by a calendar that provides real-time orientation. If a delay in treatment is experienced, the patient is able to adjust the calendar to reflect real-time events. Web links and layered educational content provide patients with quick access to information that specifically meets their needs. My CARE Source offers validated information on an individual's specific condition that assists them in seeing the full picture of their treatment journey. This is a far step from a
Through the My CARE Source portal, patients are able to document, track and monitor symptoms and/or side effects of treatment, as well as view their treatment plan according to the weeks and cycles of therapy they are receiving. This supports patients in becoming active participants in the management of their care, improving interaction with their healthcare team.

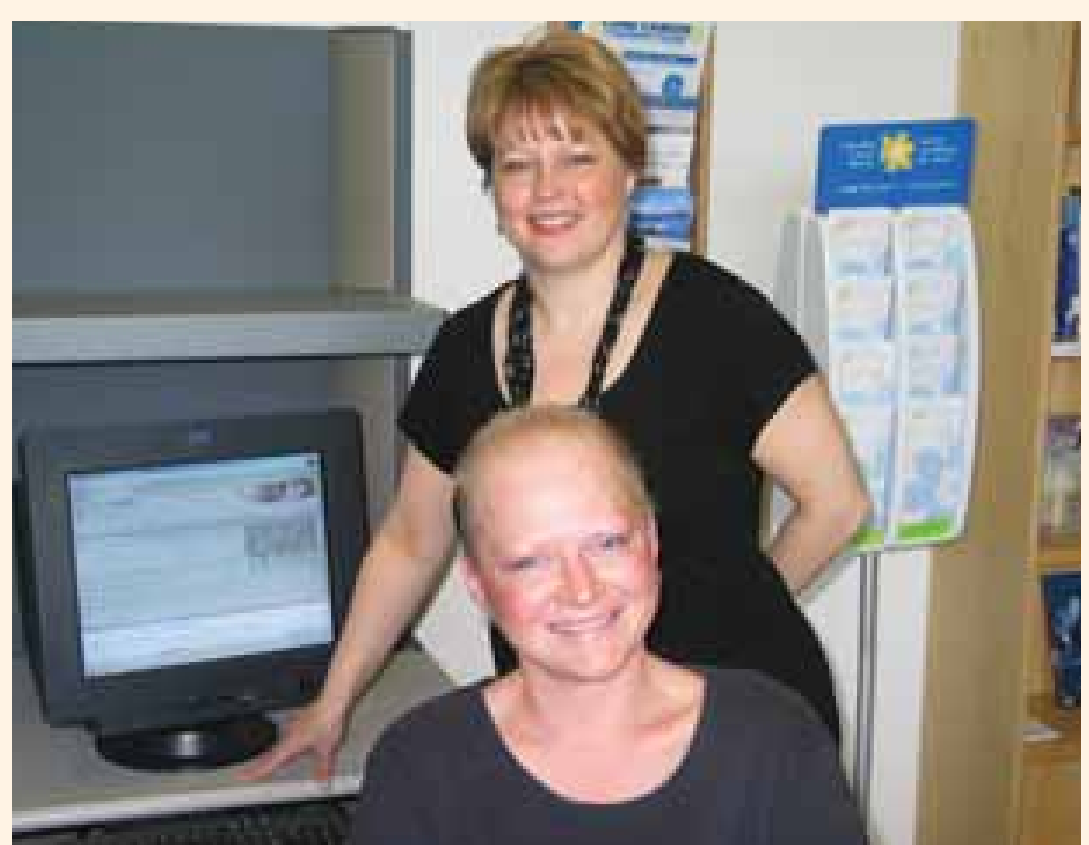

Allison Armstrong is a patient who was part of the pilot study with Claudette DeLenardo, Project Director, Patient Portal

"Google" search on "breast cancer" that yields 4,660,000 unfiltered results for a patient to sift through.
My CARE Source is accessible from any computer with Internet access, allowing patients to access their 
personal health information from anywhere - and with anyone. The program encourages patients to become involved, and to involve their loved ones in their treatment journey. Through My CARE Source, patients become their own "project manager"; they are given a tool that assists them in making informed choices about their care while also promoting communication, and giving them a sense of auton-

In addition, My CARE Source offers a health profile for the patient and family members, enables patients to request medication refills and/or transfer medication prescriptions to the hospital's pharmacy, provides patients with a space for a personal diary to track their thoughts and feelings during their treatment journey and gives them access to a care team members directory. omy and control.

Grand River Hospital has invested significantly to automate the processes that ensure patients receive their healthcare services, including the installment of Health Information Systems (HIS) that register and schedule patients, record and display their results, and hold their electronic record. The patient portal leverages the use of these existing systems.

Partnering with its Information Technology provider, McKesson Information Solutions, Grand River Hospital leveraged the core functionality of the HorizonWP Patient Portal as the base to My CARE Source. As McKesson worked to develop the software, the hospital moved forward in producing a richly cross-referenced library of health information content, specific to the educational and care management needs of its community's oncology patients.

Initial feedback from a pilot group of patients with breast cancer who have been using My CARE Source has been overwhelmingly positive. All members of the pilot group have indicated that they would recommend My CARE
Source to another person. Those involved in the development and implementation of the portal believe it will dramatically improve the patientprovider relationship, helping to promote a patientcentric culture that empowers patients to participate in their care. Hospital officials will continue to evaluate the success of My CARE Source through the analysis of userfeedback, evaluating the utilization of the portal by patients, and determining how efficient the portal is in conveying information to the patient. Once fully implemented, a number of methods will be used to evaluate outcome measures related to patient satisfaction, patient perception of control over illness, utilization, staff satisfaction and return on investment.

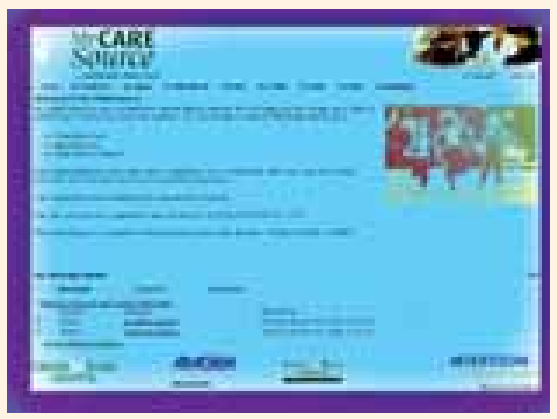

The patient's portal

The patient portal has widespread application opportunities in healthcare, in large part because it has been developed as a software application tool kit that can be customized for patients of almost any type - from cancer to diabetes and renal disease, and across the spectrum to childbirth and paedi- atrics. While Grand River Hospital intends to initially expand the portal for prostate, gastrointestional and lung cancer applications, it will ultimately move forward with adapting the tool kit for patients diagnosed with diabetes and with renal disease.

The hospital hopes that My CARE Source will help patient outcomes improve - specifically reducing the need for inpatient admissions, decreasing length of hospital stay, reducing the amount of urgent calls to the triage nurse and increasing medication compliance. In addition, My CARE Source provides the hospital with opportunity to enhance operational efficiencies and creates new revenue-generating opportunities through corporate sponsorships and by providing patients retail opportunities such as prescription transfers, medication refills and product ordering through the hospital's retail pharmacy.

The opportunities for the My CARE Source application are endless. The hospital anticipates expanding the portal to enable patients to access their lab results and view their electronic health record on-line. By providing an innovative way for patients to take an active role in the management of their care, the hospital can ultimately improve patient experiences.

For further information contact Claudette DeLenardo, Program Director, Patient Portal at Claudette.delenardo@grhosp.on.ca or call her at (519) 749-4300 ext. 5359. You can also contact Glen Kearns, Vice President and Chief Information Officer at Glen.kearns@grhosp.on.ca or call him at (519) 749-4300 ext. 2755 